\title{
ASO Visual Abstract: Optimizing Axillary Management in Clinical T1-2NO Mastectomy Patients with Positive Sentinel Lymph Nodes
}

\author{
Olga Kantor, MD MS ${ }^{1,2}$, Jessica Means, MD ${ }^{1}$, Samantha Grossmith, $\mathbf{B A}^{1}$, Tanujit Dey, $\mathrm{PhD}^{\mathbf{3}}$, \\ Jennifer R. Bellon, $\mathrm{MD}^{4}$, Elizabeth A. Mittendorf, $\mathrm{MD} \mathrm{PhD}^{1,2}$, and Tari A. King, $\mathrm{MD}^{1,2}$ \\ ${ }^{1}$ Division of Breast Surgery, Department of Surgery, Brigham and Women's Hospital, Boston, MA; ${ }^{2}$ Breast Oncology \\ Program, Dana-Farber/Brigham and Women's Cancer Center, Boston, MA; ${ }^{3}$ Center for Surgery and Public Health, \\ Department of Surgery, Brigham and Women's Hospital, Boston, MA; ${ }^{4}$ Department of Radiation Oncology, Dana-Farber \\ Cancer Institute and Brigham and Women's Hospital, Boston, MA
}

Initiation of a clinical protocol to optimize axillary management of patients with cT1-2N0 breast cancer undergoing mastectomy with one to two positive sentinel lymph nodes has decreased overtreatment of the axilla without compromising outcomes in this patient population (https://doi.org/10.1245/s10434-021-10726-3).
FUNDING J.M. acknowledges the Pamela and Nick Gelsomini Breast Surgical Oncology Fellowship Fund for support.

DISCLOSURE T.A.K. reports speakers honoraria and advisory board participation for Exact Sciences (formerly Genomic Health); and Faculty, PrecisCA cancer information service. E.A.M. reports scientific advisory board participation for Exact Sciences (formerly Genomic Health).

\section{Optimizing Axillary Management in Clinical T1-2N0 Mastectomy Patients With Positive Sentinel Lymph Nodes}

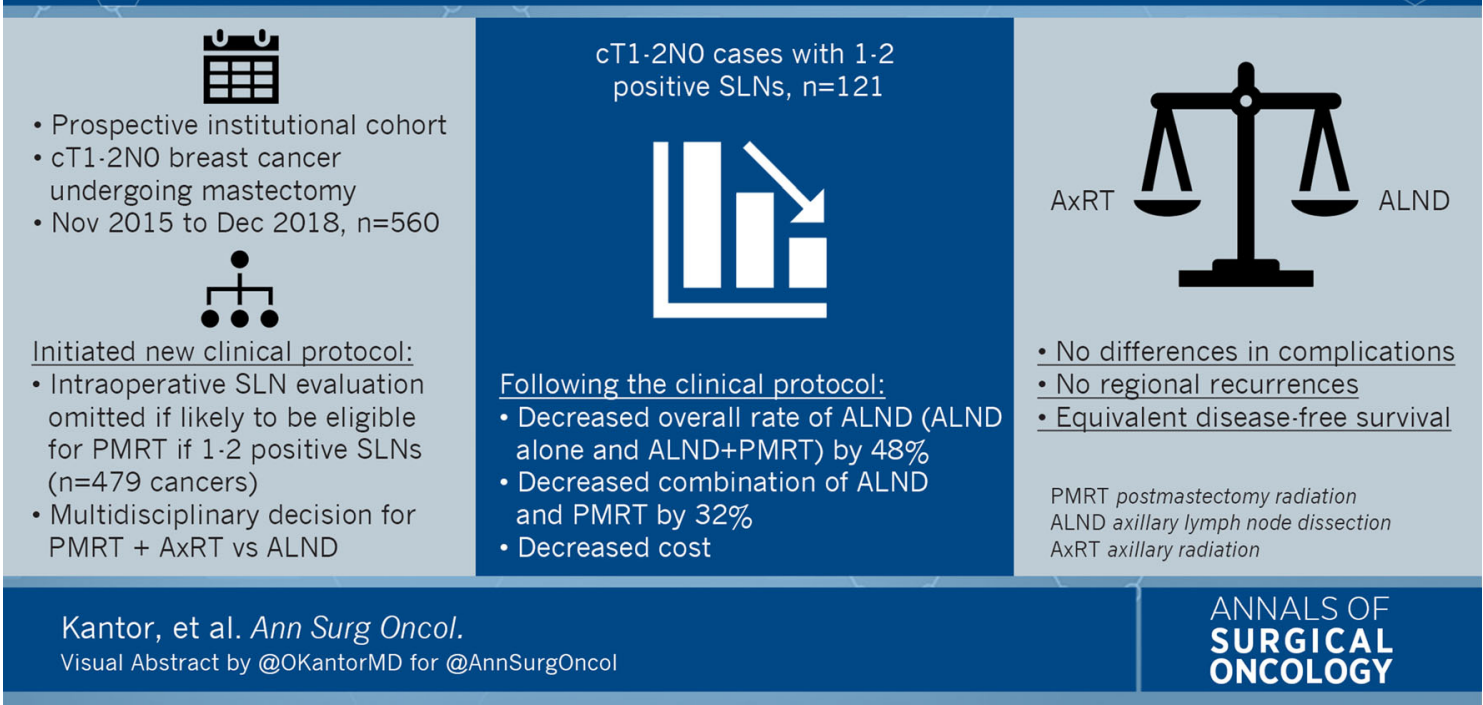

Olga Kantor and Jessica Means have contributed equally.

(C) Society of Surgical Oncology 2021

Published Online: 8 September 2021

T. A. King, MD

e-mail: tking7@bwh.harvard.edu
Publisher's Note Springer Nature remains neutral with regard to jurisdictional claims in published maps and institutional affiliations. 